\title{
Sleep and breathing: actual insight and perspectives
}

\author{
W.A. De Backer*
}

The prevalence of reported sleep disturbance in the general population is high. Sleep disturbance is often due to breathing disorders, such as various forms of sleep apnoea. In a recent European study performed in four centres, 5\% of the males and $2-3 \%$ of the females reported snoring occurring every night. Daytime sleepiness, every day, was reported by about $5 \%$ [1]. These data are impressive taken into account the likelihood of underreporting in some specific populations [2]. Objective measurements, using polysomnography in a large population, have shown an apnoea-hypopnoea index (AHI) score of 5 or higher in $9 \%$ of females and $24 \%$ of males. Two percent of females and $4 \%$ of males in the middle-age group meet the minimal criteria for the sleep apnoea syndrome (AHI $>5$ and daytime hypersomnolence) [3]. These sleep-related breathing disorders are not only more frequent than initially thought, they are also responsible for excess mortality [4] and morbidity $[1,5]$. Therefore, we should probably enhance our efforts to improve the treatment and outcome of this disease. In order to do so, we still need optimal insight into the pathophysiological mechanisms involved in the pathogenesis of apnoeas (both obstructive and central) during sleep and in the occurrence of various complications. All treatment modalities need to be evaluated carefully and with criticism. In the following issues of the European Respiratory Journal, actual insights into these topics will be summarized and put into perspective in a review series on "Sleep and Breathing".

The pathophysiological mechanisms involved in obstructive sleep apnoea (OSA) will be overviewed by W. McNicholas and P. Deegan. Factors predisposing to the disease are summarized. A careful review of the literature on the evidence of a reduced upper airway calibre in obstructive sleep apnoea will be given, together with the usefulness of several methods available for measuring upper airway calibre. Abnormalities of upper airway muscle function and co-ordination between diaphragmatic contractions and upper airway muscle activations will be discussed. The paper ends with the role of upper airway reflexes and respiratory control mechanisms in the pathogenesis of OSA, and the interfering role of the arousal mechanism in aggravating the apnoea syndrome.

Central sleep apnoea (CA) should be considered as the end of a spectrum. Instability in the breathing pattern is the main underlying mechanism and is due to the interaction of many factors. Decreased chemical drive and/or failing respiratory muscle function are associated with CA

*Correspondence: Dept of Pulmonary Medicine, University of Antwerp (UIA), Universiteitsplein 1, 2610 Wilrijk-Antwerpen, Belgium. and also with ongoing hypoventilation during wakefulness. Also, upper airway reflexes can inhibit central respiratory drive. Mostly, however, central sleep apnoea occurs as the hallmark of unstable breathing during sleep brought about by an overall increase in loop gain and the unmasking of a $\mathrm{CO}_{2}$ threshold. These aspects will be discussed in our contribution to this review series. Also, the role of arousal in aggravating the syndrome will be discussed. Micro electroencephalographic (EEG) arousals are often observed as a consequence of CA. They are responsible for sleep fragmentation and hypersomnolence during the day. CA can also occur in specific pathologies, such as chronic heart failure and (post-traumatic) brain lesions. We will end our contribution by discussing the possible treatment strategies, which are remarkably few in number. The use of nasal ventilation is well-established in CA due to respiratory muscle failure or absence of chemical drive. CA of different origin is difficult to treat with this method since most patients will not tolerate these more invasive therapies. Drug treatment, especially with acetazolamide, is easier to perform and can be promising in well-selected patients. Again, stimulation of the upper airway reflexes, as we pointed out previously [6], remains to be evaluated, but is likely to become more important in the therapy of CA.

From the clinical point of view, the manifestations and consequences of OSA are most important; and J.H. Peter will review this topic. He will address the perspectives and possibilities of early recognition of sleep-induced upper airway obstruction (UAO) and its interaction with arterial hypertension, other cardiovascular disorders, daytime sleepiness and pathophysiological mechanisms of sleep fragmentation. This will be done based on a review of the literature as well as on his own experience during the last $15 \mathrm{yrs}$, including more than 10,000 patients diagnosed and treated with sleep-related UAO. He will also discuss the fact that clinical practice has shown that parameters such as apnoea or respiratory disturbance index are not sufficient to assess the severity of the disease. Recognition and intervention by trained and experienced sleep physicians can prevent the development and manifestations of consequences of UAO in these patients.

The possible treatment strategies will be overviewed by G. Liistro, G. Aubert and D. Rodenstein. The treatment of patients with sleep apnoea should aim to achieve several objectives: alleviation of symptoms, decrease in morbidity and decrease in mortality. In addition, sideeffects should be considered. The effects on morbidity and mortality have, however, not been fully described for all therapies. General measures, such as weight loss, 
avoidance of alcohol and hypnotics, and position training will be discussed. The surgical management of obstructive sleep apnoea will be critically reviewed. It will be stressed that most series do not report sufficient data to verify the effects of surgery on sleep [7, 8]. Also, the effects on long-term survival are still to be established [9]. Some aspects of nasal continuous positive airway pressure (nCPAP) are also discussed with emphasis on the problems of compliance [10].

Sleep-related breathing disorders are symptomatic diseases associated with morbidity and mortality. Much has been learned about pathophysiology, clinical presentations and possible treatment strategies. It remains, however, crucial for the physicians in charge of the patients to recognize the disease and to have as full an insight into this pathology as possible, in order to assure adequate diagnosis and treatment. We hope that this review series will be able to contribute to this end and, therefore, be of some help in improving the well-being of our patients.

\section{References}

1. Janson C, Gislason T, De Backer W, et al. Daytime sleepiness, snoring and gastroesophageal reflux among young adults in three European countries. J Intern Med 1995; (in press).
2. Redline S, Kump K, Tishler P, et al. Gender differences in sleep-disordered breathing in a communitybased sample. Am J Respir Crit Care Med 1994; 149: 722-726.

3. Young T, Palta M, Dempsey J, et al. The occurrence of sleep-disordered breathing among middle-aged adults. $N$ Engl J Med 1993; 328: 1230-1235.

4. He J, Kryger M, Zorick F, et al. Mortality and apnea index in obstructive sleep apnea. Chest 1988; 94: 9-14.

5. Fletcher E. The relationship between systemic hypertension and obstructive sleep apnea: facts and theory. Am J Med 1995; 98: 118-128.

6. De Backer W. Upper airway reflexes and obstructive sleep apnoea. Eur Respir J 1993; 6: 12-13.

7. Rodenstein D. Assessment of uvulopalatopharyngoplasty for the treatment of sleep apnea syndrome. Sleep 1992; 15: S56-S62.

8. De Backer W, Van de Heyning P. Is the role of UPPP in nonapnoeic snorers underestimated? Eur Respir $J$ 1994; 7: 843-844.

9. Keenan S, Burt H, Ryan F, et al. Long-term survival of patients with obstructive sleep apnea treated by uvulopalatopharyngoplasty or nasal CPAP. Chest 1994; 105: 155-159.

10. Engleman H, Martin S, Deary I, et al. Effect of continuous positive airway pressure treatment on daytime function in sleep apnoea/hypopnoea syndrome. Lancet 1994; 343: 572-575. 